\title{
Gastric Occlusion due to the Intragastric Balloon Displacement in the Era of Coronavirus Disease-19 (COVID-19) Pandemic, Operative Management: a Case Report
}

\author{
Giuliano Riccardo Sarro ${ }^{1}$. Virginia Ceccarossi ${ }^{1}$ (D) Elisa Arborio ${ }^{1} \cdot$ Marco Bindi $^{1} \cdot$ Vincenzo Tripodi $^{1}$. \\ Umberto Rivolta $^{1}$ - Giuseppe Ursini ${ }^{2}$ - Matteo Marconi ${ }^{1}$
}

Received: 26 April 2020 / Revised: 26 May 2020 / Accepted: 28 May 2020 / Published online: 5 June 2020

(C) Springer Science+Business Media, LLC, part of Springer Nature 2020

\section{Introduction}

Obesity is one of the most widespread health problems worldwide. Intragastric balloons (IGB) are considered safer, miniinvasive treatments to induce weight loss $[1,2]$ in obese patients with a body mass index $(\mathrm{BMI})>30$. As other miniinvasive surgical treatments, the use of IGB is not free from possible complications.

We report a case of a patient who underwent IGB removal due to bowel obstruction in the era of SARS-CoV-2 pandemic.

\section{Case Report}

We present a case of a 44-year-old woman who underwent Bioenteric Intragastric Balloon (BIB) removal because of its migration into the gastric antrum, causing occlusion with recurrent vomiting, in the era of SARS-CoV-2 (severe acute respiratory syndrome-coronavirus-2) pandemic [3]. The patient had undergone BIB positioning for obesity 5 months earlier (October 2019). Early and late postoperative periods were uneventful. The patient had a past history of hypothyroidism due to Hashimoto disease. The body weight at the moment of the BIB placement was $74 \mathrm{~kg}$ with a BMI of 30.5 (maximum BMI was 34); she achieved a 10-kg weight loss, reaching a BMI of 26.5. In March 2020, she was admitted to Emergency Department (ED) as the subject was unable to ingest any solids or liquids for 2 weeks and vomited repeatedly. She was hemodynamically stable and had fever and cough. Neither leucocytosis nor C-reactive protein (CRP) increase was shown from blood tests, but an initial electrolyte disorder was detected. She complained a moderate abdominal pain and a palpable mass was detected in right upper quadrant during physical examination. Suspecting a BIB-related complication, she underwent chest X-rays showing a single airfluid level in the left upper quadrant and an early stage interstitial lung involvement. Abdominal ultrasonography revealed the dislocation of the device into the gastric antrum with a lot of gastric contents into the fundus and the gastric body (Fig. 1). Thus, she was admitted in surgery ward. Before the hospitalization, in this particular historical context, the patient was submitted to naso-pharyngeal test for SARS-CoV-2 disease, which resulted positive. Considering her clinical conditions and symptoms conditioning the hospitalization, the

\author{
Virginia Ceccarossi \\ vceccarossi@gmail.com \\ Giuliano Riccardo Sarro \\ giuliano.sarro@asst-ovestmi.it \\ Elisa Arborio \\ arborioelisa83@gmail.com \\ Marco Bindi \\ marcobindimd@gmail.com \\ Vincenzo Tripodi \\ vincenzo.trip@gmail.com
}

\author{
Umberto Rivolta \\ umberto.rivolta@asst-ovestmi.it \\ Giuseppe Ursini \\ giuseppe.ursini@asst-ovestmi.it \\ Matteo Marconi \\ matteo.marconi@asst-ovestmi.it
}

1 General and Oncology Surgery Department, Ospedale Giuseppe Fornaroli - Asst Ovest Milanese, Via al Donatore di Sangue, 50, 20013 Magenta, MI, Italy

2 Gastroenterology and Endoscopy Department, Ospedale Giuseppe Fornaroli - Asst Ovest Milanese, Magenta, MI, Italy 


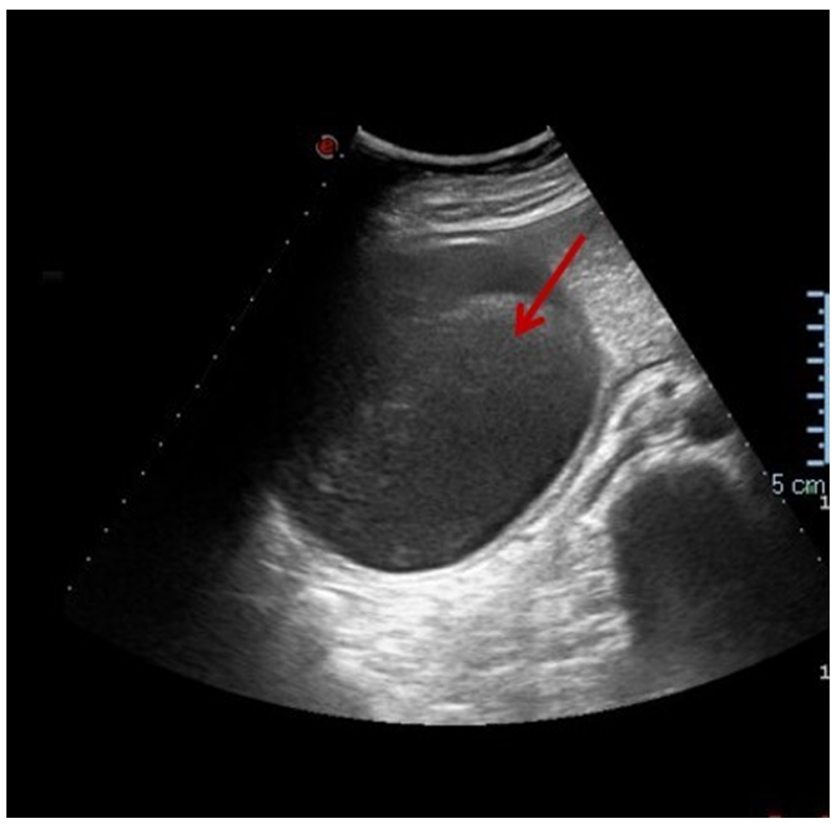

Fig. 1 Abdomen ultrasonography: the IGB is displaced into the gastric antrum (arrow)

dehydration, the electrolyte imbalance, fever and the potential evolution into an urgent clinical situation, it was decided to submit the patient to the endoscopic removal of the BIB despite resulting positive to the viral infection, according to the British Society of Endoscopy on activity during COVID-19 [4]. Given the gastric occlusion, this patient required an urgent endoscopy procedure according to the American College of Surgeons and the European Society of Gastrointestinal Endoscopy recommendations on surgical and endoscopic procedures during COVID-19 pandemic [5, 6]; she was monitored daily and assessed with clinical and laboratory examinations to perform the endoscopic procedure. During the period of hospitalization and before the endoscopy, the patient was submitted to an upper GI oral contrast challenge that showed the displacement of the intragastric balloon into gastric antrum stopping the transoral contrast from proceeding through the gastric lumen (Fig. 2). The day after, an urgent esophagogastroduodenoscopy (EGD) was performed in order to remove the $\mathrm{BIB}$ under general anaesthesia. The procedure was performed according to the recommendations for treatment of COVID-19 patients in force in that moment (negativepressure operating room, double gloves, disposable hairnet, protective eyewear, waterproof gowns, FFP3 masks, endoscopy unit cleaning with virucidal agents after the procedure) $[4,5,7-9]$. Infection prevention and control have been shown to be effective in assuring the safety of both health care professionals and patients during both emergency procedures, surgical and endoscopic [6]. The intragastric device, still inflated with $500 \mathrm{~cm}^{3}$ of saline solution and $2 \mathrm{~cm}^{3}$ of methylene blue dye, was completely deflated and removed endoscopically (Fig. 3a, b and c). After the procedure, an

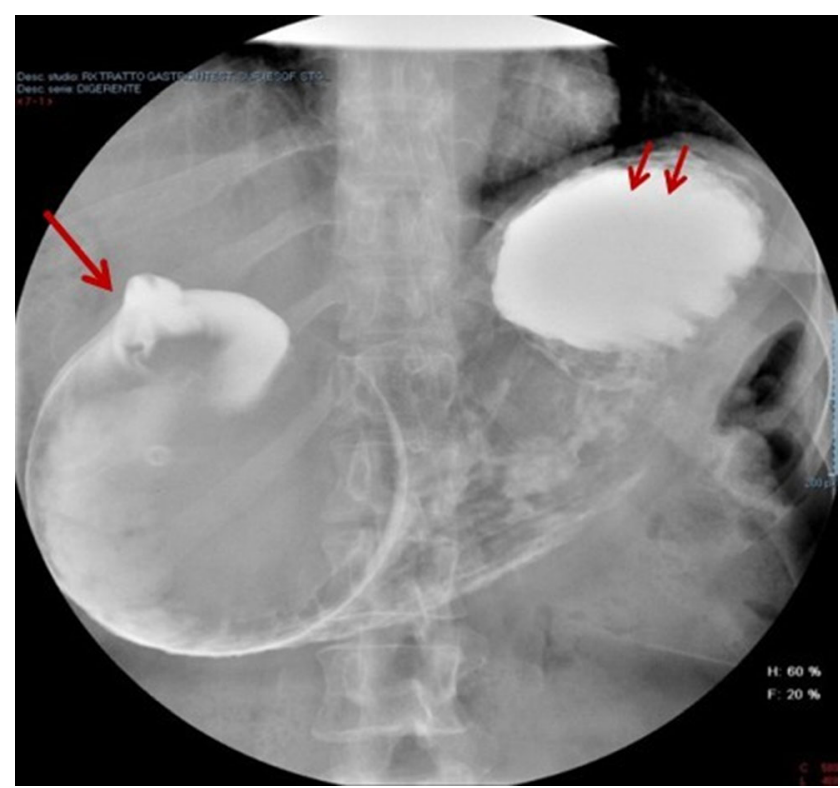

Fig. 2 Upper gastrointestinal tract radiography with gastrographin; the image shows the displacement of the IGB (single arrow) into the gastric lumen and lots of gastric contents in the fundus (double arrow)

endoscopic control was performed, and no esophagogastric injuries were detected. The postoperative period was uneventful. During the hospitalization, the patient was isolated; she did not suffer from nausea or vomiting. Intravenous hydration was initially set until normal electrolyte balance was restored and oral intake of fluids and solids gradually restarted. Despite the viral infection, since she was afebrile with no cold or cough, she was discharged with the indication to remain in quarantine until next test for SARS-CoV-2.

\section{Discussion}

The COVID-19 outbreak, caused by the coronavirus SARS$\mathrm{CoV}-2$, has emerged as a rapidly spreading disease affecting more than 100 countries in the world, being declared as pandemic by the World Health Organization (WHO) [3]. The disease is primarily spread through large respiratory droplets, but other routes of transmission have been identified, such as stools of affected people. Main symptoms are fever, dry cough, fatigue and also sore throat, headache, diarrhoea and myalgias [10]. As seen, the infection can rapidly develop into a severe acute respiratory distress syndrome that can cause death.

Recent studies reported that comorbidities may represent risk factors for adverse outcomes during this infectious disease. As Yang suggests in his meta-analysis, hypertension, diabetes, cardiovascular diseases, and respiratory diseases were present in COVID-19-infected patients, with a prevalence of $17 \%, 8 \%, 5 \%$ and $2 \%$, respectively, being also related to a more severe clinical presentation [11]. 
Fig.3 a-c The sequence of the IGB endoscopic removal. a IGB inflated into the gastric antrum. b Deflation of the IGB. $\mathbf{c}$ Removal of the IGB completely deflated
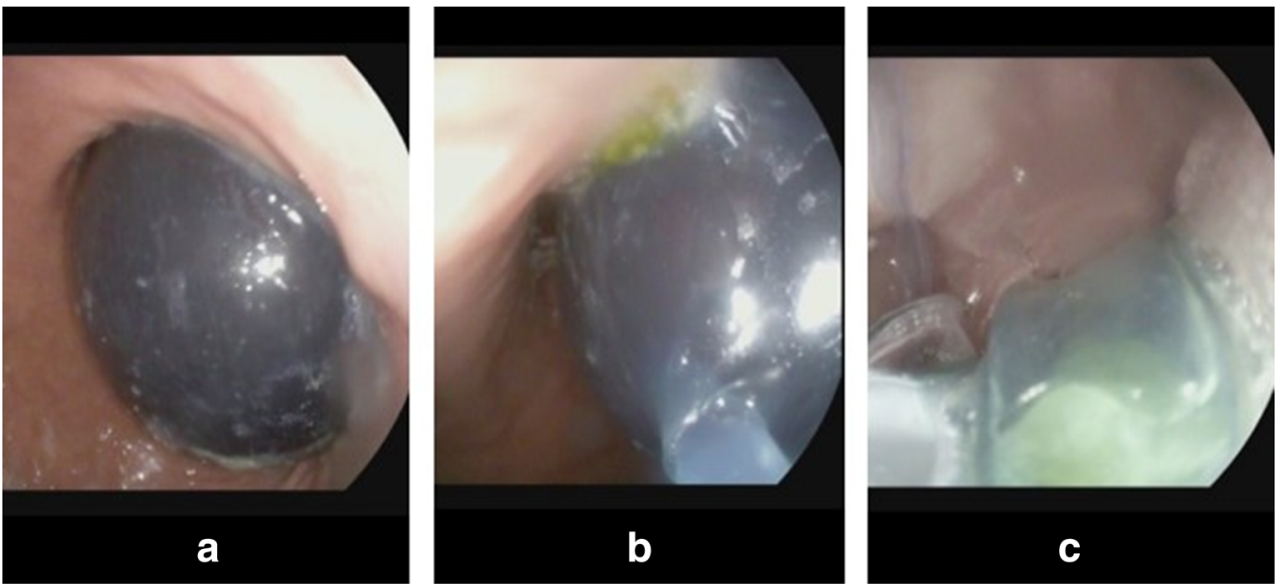

The role of overweight and obesity as potential risk factors of a severe COVID-19 is still unknown. Currently, there is no scientific evidence as to whether BMI may influence the evolution of this infectious disease or be responsible of potentially fatal consequences of it, especially if the patient, as in our case presented above, is facing an emergency clinical situation requiring urgent endoscopic or surgical procedures. In these cases, it would be necessary to recognize the situation of emergency and what could be the consequences of a non-operative management or of a delayed operative treatment on clinical outcome of the patient. The decision-making and all of treatments should be discussed in a multidisciplinary context and tailored on patient clinical pathological characteristics.

Regarding this particular topic, we searched in the literature if overweight and obesity could be considered risk factors for severe viral infectious diseases and if they could influence the clinical outcome of ill patients. We found some evidence regarding the Middle East Respiratory Syndrome (MERS), responsible for a severe respiratory syndrome in 2012 in the Middle East, caused by a coronavirus (MERS-CoV), and regarding the seasonal and pandemic influenza.

In their review about MERS, Badawi et al. observed that $16 \%$ of the cases analysed were affected by obesity. They suggested that there was an increased risk of developing severe MERS complications in people with chronic diseases, such as hypertension, diabetes and obesity, similarly noted also for viral influenza and related complications. Moreover, they highlighted the fact that metabolic chronic diseases such as diabetes, hypertension, cardiovascular diseases and obesity could be linked etiologically to the pathogenesis of MERS, sharing several common features with viral infectious disorders and their complications, such as endothelial dysfunction, a proinflammatory state with cytokines activation, an attenuation of the innate immune response [12]. Some of these immunological features and the dysregulation of the immunological response have been reported in other severe viral infections and also in the COVID-19 disease [13, 14].
Besides, regarding the severe pandemic influenza, Mertz et al. noted that, compared with people with no comorbidities, the viral disease occurred more often in those who were obese (odds ratio (OR) for mortality of $2.74,95 \%$ CI 1.56-4.80). Obesity not only increased the risk of death but was significantly associated with the need for admission to hospital or to intensive care unit [15].

On the basis of this evidence, we could assume that similar mechanisms could be the basis of COVID-19 and that overweight or obesity, as other metabolic conditions, could represent an additional risk factor in determining the severity of clinical manifestations of this infectious disease, especially in emergency situations that require fast operative management. In these cases, we can assume also that, if treatment were delayed, overweight patients could present a worse development of both emergency condition and infectious disease. Further data and evidence are surely needed in support of this hypothesis.

Regarding the management of COVID-19 patients requiring surgical or endoscopic treatments, some recommendations have been proposed by the American College of Surgeons, the European Society of Gastrointestinal Endoscopy and the British Society of Endoscopy [4-7, 9]. Regarding patients who should undergo surgery, authors firmly suggest that, as the surgical condition persists, it is important to recognize that the decision to delay or perform the surgical procedure must be made considering several aspects, both medical and logistical. They affirm that, given the uncertainty regarding the impact of COVID-19 over the next months, delaying surgical treatments is a risk and could lead to more severe emergencies at a time when they will be more difficult to manage [5].

Risk of infection by SARS-CoV-2 during endoscopy procedures is increased from inhalation of airborne droplets, conjunctival contact and stools contamination for both health care professionals and for patients. Because of this, endoscopists had to implement measures for infection prevention and control, with a detailed protocol including a management strategy, 
risk stratification, correct use of the personal protective equipment (PPE) and selected interventions for patients at high risk of COVID19. If patient requires an urgent endoscopic procedure, authors recommend the importance of a risk stratification for symptoms of COVID-19 that should be done prior to endoscopy and at the time of endoscopy; this attitude allows all the health care professionals involved in the case to decide and prepare every device needed for safety procedure $[4,6]$.

The medical need for a given procedure should be certainly established by a surgeon or endoscopist with direct expertise to determine what medical risks will be incurred in case of delay. The logistical feasibility for a given procedure should be determined by administrative personnel, considering the hospital and community resources [16].

The risk for the patient should include a daily assessment of the real risk of proceeding and the real risk caused by the delay. Although COVID-19 is a clear risk to all, it is mostly a risk for patients requiring surgical and urgent care and for patient with comorbidities. Therefore, both surgical and endoscopic procedures should be considered not only based on risks associated to COVID-19 but also considering all medical and logistical information on the case [5].

How to perform surgery and treat patients requiring surgical treatment has become an urgent problem in this particular historical era of SARS-CoV-2 pandemic. A multidisciplinary diagnosis and management are recommended to achieve compliance with the epidemic context. For patients undergoing emergency surgery, a cooperation should be important to alert the operating room, the anaesthesiologist and nursery team to decide and assure the safest approach to the patient [16].

\section{Compliance with Ethical Standards}

Conflict of Interest The authors declare that they have no conflict of interest.

Ethical Approval All procedures performed in studies involving human participants were in accordance with the ethical standards of the institutional and/or national research committee and with the 1964 Helsinki declaration and its later amendments or comparable ethical standards.

Informed Consent Informed consent was obtained from all individual participants included in the study.

\section{References}

1. Yorke E, Switzer NJ, Reso A, et al. Intragastric balloon for management of severe obesity: a systematic review. Obes Surg. 2016;26(9):2248-54.
2. Tate CM, Geliebter A. Intragastric balloon treatment for obesity: review of recent studies. Adv Ther. 2017;34(8):1859-75.

3. World Health Organization Rolling updates on coronavirus disease (COVID-19). 2020. Available from: https://www.who.int/ emergencies/diseases/novel-coronavirus-2019/events-as-theyhappen.

4. British Society of Endoscopy COVID-19 guidance and advice - GI endoscopy activity and COVID-19: next steps - Updated 03.04.20. Available from: https://www.bsg.org.uk/covid-19-advice/giendoscopy-activity-and-covid-19-next-steps/.

5. American College of Surgeons COVID-19: Guidance for triage of non-emergent surgical procedures 2020 (March 17, 2020). Available from: https://www.facs.org/covid-19/clinical-guidance.

6. European Society of Gastrointestinal Endoscopy ESGE and ESGENA Position Statement on gastrointestinal endoscopy and the COVID-19 pandemic. Available from: https://www.esge.com/ esge-and-esgena-position-statement-on-gastrointestinalendoscopy-and-the-covid-19-pandemic/.

7. American Society of Anesthesiologists (ASA) COVID-19 resources for anesthesiologists COVID-19 information for health care professionals 2020. Available from: https://www.asahq.org/aboutasa/governance-and-committees/asa-committees/committee-onoccupational-health/coronavirus.

8. World Federation of Societes of Anesthesiologists (WFSA) Coronavirus - guidance for anaesthesia and perioperative care providers (2020). Available from: https://www.wfsahq.org/latestnews/latestnews/943-coronavirus-staying-safe.

9. Society of Gastrointestinal and Endoscopic Surgeons - European Association for Endoscopic Surgery EAES and SAGES recommendations regarding surgical response to COVID-19 crisis, March 2020. Available from: https://www.sages.org/ recommendations-surgical-response-covid-19/.

10. Gupta R, Ghosh A, Singh AK, et al. Clinical considerations for patients with diabetes in times of COVID-19 epidemic. Diabetes Metab Syndr. 2020;14(3):211-2.

11. Yang J, Zheng Y, Gou X, Pu K, Chen Z, Guo Q, et al. Prevalence of comorbidities in the novel Wuhan coronavirus (COVID-19) infection: a systematic review and meta-analysis Int J Infect Dis. 2020.

12. Badawi A, Ryoo SG. Prevalence of comorbidities in the Middle East respiratory syndrome coronavirus (MERS-CoV): a systematic review and meta-analysis. Int J Infect Dis. 2016;49:129-33.

13. Rothan HA, Byrareddy SN. The epidemiology and pathogenesis of coronavirus disease (COVID-19) outbreak. J Autoimmun. 2020;109:102433.

14. Xu Z, Shi L, Wang Y, et al. Pathological findings of COVID-19 associated with acute respiratory distress syndrome. Lancet Respir Med. 2020;8(4):420-2.

15. Mertz D, Kim TH, Johnstone J, et al. Populations at risk for severe or complicated influenza illness: systematic review and meta-analysis. BMJ. 2013;347:f5061.

16. Tao KX, Zhang BX, Zhang P, et al. Recommendations for general surgery clinical practice in 2019 coronavirus disease situation. Zhonghua Wai Ke Za Zhi. 2020;58(3):170-7.

Publisher's Note Springer Nature remains neutral with regard to jurisdictional claims in published maps and institutional affiliations. 